\title{
A Internet e a busca da informação em comunidades científicas: um estudo focado nos pesquisadores da UFSC
}

Marili Isensee Lopes

\author{
Mestre em Ciência da Informação - UFSC; \\ Bibliotecária da Biblioteca Universitária da UFSC
}

Edna Lúcia da Silva

\begin{abstract}
Doutora em Ciência da Informação - UFRJ-IBICT; Professora do Departamento de Ciência da Informação e do Programa de Pós-graduação em Ciência da Informação UFSC
\end{abstract}

Pesquisa que teve por objetivo verificar o uso da Internet no processo de busca da informação em comunidades científicas, visando detectar a ocorrência da desintermediação da informação e suas conseqüências no processo de comunicação científica e na atuação das unidades de informação. Nesta pesquisa, a desintermediação foi considerada como a ausência de intermediários entre os recursos de informação e os pesquisadores. A amostra foi constituída por 324 pesquisadores-docentes da Universidade Federal de Santa Catarina, vinculados aos seus Programas de PósGraduação, selecionada em três etapas: aleatória estratificada por áreas de atuação, aleatória simples por notas de avaliação da Capes e aleatória por conglomerados por programa. Foi utilizado o questionário como instrumento de coleta dos dados, com questões voltadas para o levantamento de informações sóciodemográficas e para o uso de recursos informacionais. Os dados foram organizados por meio do pacote estatístico SPSS, versão 11.0, utilizando a estatística descritiva (freqüências e porcentagens), de associações (teste 2) e comparativas (teste de Kruskal-Wallis e Mann-Whitney), considerando-se um nível de significância de 5\%. As análises dos resultados permitiram constatar que, dentre outros, os percursos preferidos na busca da informação, por esses pesquisadores, foram a biblioteca particular, com 46,3\%, e a Internet, com 43,2\%. O periódico foi o mais indicado dentre os recursos disponibilizados no Sistema BU/UFSC. Os pesquisadores indicaram que preferem obter o material em formato eletrônico devido à 
sua acessibilidade. Em relação aos mediadores no processo de busca da informação, os colegas/pares foram os mais citados. O correio eletrônico foi o recurso da Internet mais utilizado, o que também confirma a finalidade de uso da rede, onde a comunicação entre pares, com 65,4\%, foi a que obteve maior incidência de indicações. Dentre os serviços on-line disponibilizados pela BU/UFSC, o Portal Capes foi o mais indicado (61.7\%). Quanto ao processo de desintermediação, embora tenha aumentado em todas as áreas, considerando-se que os pesquisadores estão utilizando com autonomia os recursos eletrônicos no processo de busca da informação, conclui-se que o Sistema BU/UFSC ocupa um lugar de destaque nesse processo, em função do uso significativo do acervo tradicional, bem como dos serviços on-line disponibilizados pelo sistema. Quanto aos profissionais bibliotecários, os resultados permitiram concluir que eles não são os mediadores reconhecidos como preferenciais por esta comunidade científica.

Palavras-chave: Comunicação científica; Busca da informação; Desintermediação da informação.

\section{The Internet and the information search in scientific communities: a study focused on researchers of the Federal University of Santa Catarina, Florianópolis, Brazil.}

This research attempted to verify the use of Internet in information search processes in scientific communities, aiming to detect the occurrence of information disintermediation and its consequences on scientific community process and information as well as its consequences on scientific communication process and performance on the information unit. In this research, disintermediation was considered as absence of intermediates between the information resources and researchers. A sample of 324 researchers/teaching staff of the Federal University of Santa Catarina, linked to Graduate Programs was used and sorted by: random and stratified by area of knowledge, random and simple, by 
Capes evaluation grades, and random and conglomerated by the graduate program. A questionnaire was used as the tool for collecting data with questions for the survey of sociodemographic and resource use information. The data were organized using the SPSS statistics package version 11.0 using descriptive statistics (frequencies and percentages), association statistics ( $\chi 2$ test), and comparative statistics (Kruskal-Wallis and Mann-Whitney test), at $p=0.05$. The analyses of the results allowed to confirm that pathways preferred by researchers in the search for information were: private library, $46.3 \%$, and Internet, $43.2 \%$. Among the resources available at the UL/UFSC System the journal was the one most indicated by researchers. A preference for materials in electronic format was also indicated due to the easier accessibility. In relation to the mediators in the process of information search, colleagues/pairs were the most indicated. Electronic mail was the most used Internet resource. This also confirms the use of network where the communication between pairs, with $65.4 \%$, was the indication with the highest frequency. Among the available online services at UL/UFSC, Capes Gateway was the most indicated (61.7\%). As for the disintermediation process, since it has increased in all areas because researchers are autonomously using the electronic resources in information searching, it can be concluded that UL/UFSC has a considerable position in this process since there is a significant use of the traditional collection, as well as the online system service. The results allowed to conclude that librarian workers are not recognized as preferential mediators by this scientific community.

Keywords: Scientific communication; Information searching; Information desintermediation.

Recebido em 28.06.2007 Aceito em 01.10.2007

\section{Introdução}

Para Kuhn (1992), uma comunidade científica se caracteriza pela prática de uma especialidade científica, por uma formação teórica comum, pela circulação abundante de informação no interior do grupo e pela unanimidade de juízo em assuntos profissionais.

Ao se pesquisar temas que envolvem comunidades científicas, o olhar volta-se naturalmente para as universidades, lugar dominante na formação de investigadores e de pesquisas, na maioria dos países de terceiro mundo. No Brasil, reconhece-se que mais de $80 \%$ das pesquisas 
são desenvolvidas nos centros de investigação das universidades, habitualmente vinculados a programas de formação de pós-graduação (mestrados e doutorados) (LOVISOLO, 1997).

Da mesma forma, pensar em pesquisas que envolvem comunidades científicas implica em pensar nos processos de comunicação científica desenvolvidos pelos pesquisadores das universidades. Assim, pensar em ciência também significa refletir sobre os processos que envolvem informação e comunicação, ou ainda pensar em um sistema social que "integra elementos que vão desde a figura do pesquisador/cientista/acadêmico ao fluxo de idéias, fatos, teorias, métodos, literatura científica e instrumentos que permitem a operacionalização das investigações" (TARGINO, 2000, p. 24).

Atualmente as universidades brasileiras têm como principais atividades: pesquisa, ensino e extensão. Tais atividades, segundo Castellani e Zwicker (2000), apresentam como característica comum o fato de lidarem com o conhecimento, de sua produção até sua disseminação. A ênfase dada a cada uma delas tem mudado com o tempo, influenciada pelo próprio Estado e pelas políticas públicas.

A atividade científica tem como principais objetivos a comunicação da informação e a disseminação dos conhecimentos produzidos, visando o próprio desenvolvimento e progresso da ciência. Pode-se, inclusive, afirmar que a ciência se desenvolveu largamente em torno dos documentos científicos. Ao longo da história e no mundo atual, pode-se perceber que praticamente nada mudou nesse sentido. $O$ valor das publicações é mantido para a ciência, como o permite perceber a declaração clássica de Ziman (1979), quando afirma que "ciência é conhecimento público". O que realmente mudou e vem mudando são os suportes das publicações científicas. Se antes eram as cartas, atas escritas à mão; hoje são fotocópias, faxes, e-mails e tantos outros formatos que ainda expressam os mesmos objetivos daquelas primeiras comunidades, ou seja, unir grupos de pessoas com os mesmos interesses e disseminar a informação entre seus pares (BROW; DUGUID, 2001).

A comunicação científica, segundo Meadows (1999, p. vii), "situa-se no próprio coração da ciência" e, de qualquer que seja o ângulo examinado, se constitui parte essencial do processo de investigação científica. Para Targino (1999/2000, p. 347), "a ciência faz da pesquisa científica seu instrumento mor e da comunicação científica seu elemento básico, de tal forma que a pesquisa científica e as divulgações de seus resultados são atividades inseparáveis".

O sistema de comunicação científica definido por Garvey e Griffth (GARVEY, 1979) e adotado em muitos estudos, desde os anos de 1970, foi baseado em dois tipos de canais de comunicação, o informal e o formal. A comunicação informal é usada no início do processo de pesquisa e se concretiza por meio da oralidade, através dos contatos face-a-face, ou contatos interpessoais, utilizando quaisquer recursos que não exijam certa formalidade, como nas participações em associações, colégios invisíveis, reuniões científicas, telefonemas, bem como em toda troca informal através de recursos escritos como cartas, faxes, mensagens de 
e-mails, entre outros (MEADOWS, 1999). Quando finalizado o processo de pesquisa, os canais formais são usados para a divulgação dos resultados, que se concretiza por meio de recursos informacionais disponibilizados na forma de publicações, nas fontes de informação primárias, secundárias e terciárias.

As novas tecnologias de informação e comunicação (TICs) introduziram mudanças nesse modelo de sistema de comunicação científica proposto por Garvey e Griffth (GARVEY, 1979). É importante ressaltar que, dentre as TICs, destaca-se a Internet, como o principal recurso tecnológico de comunicação e informação. Para Sullivan (2002), a Internet pode ser considerada uma metarede, pois integra várias redes que interconectam indivíduos e instituições, proporcionando um espaço de circulação de informações democrático, sem barreiras, sem hierarquias, sem intermediários e em tempo real.

No Brasil, a partir de 1992, com a instalação da espinha dorsal da Rede Nacional de Pesquisa (RNP) do Conselho Nacional de Pesquisa (CNPq), a comunicação via Internet foi introduzida. Em 1999 os ministérios da Educação e da Ciência e Tecnologia assinaram um convênio para a implantação do backbone RNP2, que passou a interligar o país com uma rede de alta tecnologia. O backbone RNP2 encontra-se atualmente constituído por uma rede acadêmica distribuída entre 27 estados da Federação brasileira, interligando 329 instituições de ensino e pesquisa, com uma capacidade de tráfego de $335 \mathrm{Mbps}$, e mantendo uma conectividade internacional com os Estados Unidos e a Europa, através de Portugal, de 202 Mbps (REDE NACIONAL DE PESQUISA, 2005).

Em Santa Catarina, o Pop-SC, um dos backbone da RNP, está fisicamente instalado, sob a coordenação do Núcleo de Processamento de Dados da UFSC. Esse ponto de presença da RNP atende às necessidades operacionais da rede, à demanda de conectividade e de informações de usuários de todo o Estado de Santa Catarina e de toda comunidade científica e administrativa, bem como ao uso acadêmico da UFSC.

Além dos pontos de acesso na instituição, o provedor, via UFSC, disponibiliza a seus usuários (alunos de graduação e pós-graduação, professores e servidores técnico-administrativos) o serviço de acesso remoto, por meio de cadastramento, como também serviços tais como: acesso discado, com protocolos de autenticação Asymmetrical Digital Subscriber Line (ADSL) ou Virtual Private Networks (VNP).

As tecnologias da informação e comunicação foram incorporadas a várias atividades do meio acadêmico. Considerando-se que as universidades são as maiores produtoras de conhecimento científico, que seus membros são atuantes no processo de criação e transmissão do conhecimento, e ainda que a informação é o insumo básico nesse processo, as atividades de pesquisa têm sido as maiores beneficiadas pela infra-estrutura existente para o uso das TICs.

Segundo Vaz (2001), as novas tecnologias de produção, processamento e difusão de informações, como o computador, os satélites, os cabos de fibra ótica e os softwares de interface, têm levado a uma redução em termos de custo e velocidade, e à facilidade de troca de 
informações, proporcionando assim mudanças no modo como as pessoas aprendem, ensinam, acessam e usam a informação, bem como a adoção de novos métodos de produzir, organizar, estocar, recuperar e disseminar a informação.

Questões como a validação dos conteúdos dos meios eletrônicos, questões autorais, de custo, controle bibliográfico, armazenagem, conservação, qualidade e consistência das informações, processo de avaliação, entre outros, vêm sendo discutidas por diversos autores, tais como Costa, Silva e Costa (2001), Nehmy e Paim (2003); Meadows (2001); Silva, Menezes e Bissani (2002) e Targino (2002).

Estudos como os de Ferreira (1995) e Pinheiro (2003) têm confirmado que a adoção das TICs no processo de comunicação científica tem crescido e vem afetando, de forma positiva, as atividades de pesquisa. Os autores são unânimes quando afirmam que as TICs propiciam maior eficiência na produção, avaliação e difusão do conhecimento, e promovem melhoria e agilidade nos resultados alcançados no campo da pesquisa e da ciência.

Nesse aspecto, a literatura, de maneira geral, tem revelado que a comunicação interpessoal vem sendo favorecida pela adoção das TICs. Diversos estudos indicam que o correio eletrônico é muito utilizado pelas comunidades científicas (ABELS; LIEBSCHER; DENMAN, 1996; CASTELLANI; ZWICKER, 2000; COSTA; SILVA; COSTA, 2001; FERREIRA, 1995; MEADOWS, 2001; PINHEIRO, 2003; SOUZA, 2003; VIEIRA, 1998). Os colégios invisíveis de Solla Price (1976) foram substituídos pelos contatos via Internet.

Além disso, diversas fontes importantíssimas para a busca de informações estão disponibilizadas on-line. Obras de referência (como, por exemplo, bibliografias, índices e resumos), antes impressas e publicadas em papel, estão sendo substituídas por bases de dados digitais. Algumas dessas bases já oferecem, além do dado referencial e resumo do documento, o seu texto integral. Paralelamente surgiram as bibliotecas virtuais, que facilitam o processo de busca e o acesso à informação pelos cientistas, pois disponibilizam documentos eletrônicos para consulta em tela ou mediante impressão.

Os repositórios digitais também fazem parte da realidade de muitos pesquisadores. Eles operam com a tecnologia e a filosofia dos arquivos abertos (open archives), e estão em processo de consolidação e reconhecimento como fontes confiáveis de informação científica. Em outubro de 2003, representantes de importantes instituições científicas européias, entre as quais a Sociedade Max-Plank (Alemanha) e o Centre National de la Recherche Scientifique (França), subscreveram a Declaração de Berlim, sobre o acesso livre ao conhecimento nas ciências e humanidades, dando apoio às iniciativas nesse sentido. Esse documento, firmado na Conference on Open Access to Knowledge in the Sciences and Humanities (2003), visou encorajar os investigadores e bolsistas a depositarem os seus trabalhos e promoverem o open access, a distribuição irrestrita, a interoperabilidade das iniciativas dos arquivos abertos e a sua preservação a longo prazo. 
Embora a literatura venha ressaltando os avanços das TICs como ferramentas poderosas nas atividades científicas, transformando-as em estruturas dinâmicas e competitivas, alguns autores fazem ponderações relacionadas às mudanças geradas por tais tecnologias nas atividades de ensino e pesquisa científica. Para Abels, Liebscher e Denman (1996), os fatores que impedem ou incentivam a adoção das TICs pelos pesquisadores estão relacionados ao sistema, referindo-se à acessibilidade (proximidade, primeira experiência, facilidade de uso e disponibilidade de equipamentos); aos fatores pessoais e profissionais (questões de comportamento na busca da informação, nas quais estão incluídas as diferenças entre áreas do conhecimento, tarefas desenvolvidas e utilidades percebidas); e aos fatores institucionais, relacionados à infraestrutura, no que se refere aos investimentos e à manutenção dos equipamentos e redes, bem como à realização de treinamentos.

Considerando o importante papel das novas tecnologias da informação, principalmente a Internet, no mundo e nas comunidades científicas, esta pesquisa objetivou focar o processo de comunicação científica, especificamente com relação ao uso da Internet na busca da informação. A pesquisa apóia-se na hipótese de que o uso da Internet por essa comunidade tem provocado mudanças no seu comportamento em relação ao acesso à informação, e no fato de que isso tem interferido no processo tradicional de busca de informação científica e vem trazendo conseqüências para as unidades de informação.

A Internet, possibilitando a divulgação de idéias e de informação por qualquer pessoa, gerou igualmente mudanças nos processos tradicionais de comunicação e modificou a relação entre os autores/editores/bibliotecas/leitores, colocando em cheque a cadeia tradicional de transferência de informação. O papel mais importante das unidades de informação e, conseqüentemente, de profissionais como os bibliotecários, até então era constituído pela ação de intermediação entre a informação produzida (publicadores) e os usuários da informação.

Para Fourie (2001), a desintermediação é entendida como a busca de informação por um usuário final, sem a necessidade do envolvimento de terceiros. Significa a eliminação do mediador entre a informação (ou qualquer produto) e seus usuários finais, o que, conseqüentemente, acarreta o que o autor denomina de "potencialização" dos usuários. Quando aplicado às unidades de informação (bibliotecas), o termo significa a evolução do acesso à informação, que se dava através de depósitos físicos centralizados, para as fontes alternativas obtidas diretamente através de computadores e redes de informação.

Assim, ficou estabelecido como objetivo geral desta pesquisa: analisar o uso da Internet no processo de busca da informação em comunidades científicas, e se sua utilização está gerando a desintermediação da informação. Como objetivos específicos: caracterizar o perfil sóciodemográfico dos pesquisadores; identificar o percurso que fazem para obter informação; identificar os recursos eletrônicos mais 
utilizados pelos pesquisadores, bem como a freqüência e as barreiras para o uso da Internet; verificar a existência de associação entre as características do perfil dos pesquisadores e o uso de recursos eletrônicos; detectar a ocorrência do fenômeno da desintermediação no processo de busca da informação; comparar a ocorrência da desintermediação da informação no processo de busca da informação, considerando-se as áreas de atuação dos pesquisadores.

Pressupõe-se que a análise desse processo irá permitir o entendimento de como as TICs estão interferindo e contribuindo para o acesso à informação na UFSC, o que possibilitará o melhor direcionamento de recursos e das políticas de desenvolvimento de coleções e de implantação de serviços nas unidades de informação dessa universidade, para ajustá-los em função dos comportamentos, necessidades e problemas detectados.

\section{Procedimentos metodológicos}

A pesquisa desenvolvida caracterizou-se como transversal descritiva e comparativa, (GIL, 2002; RICHARDSON et al., 1999), com uso de técnicas quantitativas e qualitativas para a abordagem do problema. Classifica-se como pesquisa transversal por ter sido realizada em um único instante no tempo, obtendo-se assim um recorte momentâneo do fenômeno investigado. É descritiva porque se propõe a descrever aspectos amplos da comunidade científica da UFSC, no que diz respeito às suas características sócio-demográficas, aos meios adotados para obter a informação, bem como ao uso de recursos informacionais tradicionais e/ou on-line, no intuito de poder identificar seu comportamento em função do uso da Internet no processo de busca da informação e, a partir daí, verificar a existência do fenômeno desintermediação. Segundo Richardson et al. (1999, p. 71), pesquisas deste tipo procuram explicar fenômenos ao analisar o papel das variáveis que, de certo modo, influenciam ou causam o aparecimento dos fenômenos.

Os estudos de natureza descritiva propõem investigar o "que é", ou seja, descobrir as características de um fenômeno como tal. Nesse sentido, são considerados como objeto de estudo uma situação, um grupo ou um indivíduo.

E finalmente é comparativa, pois pretende comparar as áreas de conhecimento para constatar as diferenças com relação ao fenômeno da desintermediação da informação.

Para a delimitação da abrangência desta pesquisa, tornou-se necessário estabelecer e definir os aspectos principais envolvidos nas questões colocadas, que são: Como os recursos informacionais disponibilizados na Internet têm modificado o comportamento de busca da informação dos pesquisadores da UFSC? O uso da Internet tem levado à desintermediação no processo de busca da informação? 
Nessa perspectiva, foi necessário definir os termos apresentados a seguir:

comunicação científica: Segundo Garvey (1979), o processo de comunicação científica envolve desde a produção até a disseminação e o uso da informação. Esta pesquisa concentrou-se nas etapas iniciais do processo, mais especificamente na etapa da produção e no processo referente à busca da informação.

1. unidades de informação: São organizações sociais sem fins lucrativos, cuja função é prestar serviços de informação tangíveis e intangíveis para indivíduos e para a sociedade, conforme Tarapanoff, Araújo Junior e Cormier (2000). Nesta pesquisa, as unidades de informação foram representadas pelas bibliotecas que compõem o Sistema de Bibliotecas da UFSC.

2. recursos informacionais disponibilizados pela Internet: Foram considerados, para esta pesquisa, os recursos informacionais disponibilizados eletronicamente via Web, ftp e e-mail, sendo que, em cada um deles, foram consideradas as seguintes fontes: 1) na Web e ftp: artigos disponibilizados em periódicos eletrônicos, newstetters, e outros seriados eletrônicos, eprints, documentos originais disponibilizados por autores em páginas pessoais, relatórios técnicos, artigos de conferências, dissertações e teses, livros e outros recursos eletrônicos disponibilizados via Internet. 2) e-mail e mensagens postadas em listas de discussão, os newsgroups.

3. processo de busca da informação: Aqui definido como sendo as etapas, modos e meios usados para a localização da informação necessária aos pesquisadores para a realização de suas tarefas acadêmicas (projetos de pesquisa, estudos, atualização profissional, ensino utilizando recursos, sejam estes na forma impressa, eletrônica ou humana).

4. desintermediação: ausência de mediação de terceiros no processo de busca da informação.

A pesquisa foi realizada na Universidade Federal de Santa Catarina (UFSC). A realização da pesquisa, que envolveu diretamente os pesquisadores envolvidos nos 50 programas de pós-graduação, abarcou 50 cursos de mestrado e 34 cursos de doutorado. Como o foco da pesquisa foi o processo de busca da informação, naturalmente também envolveu as unidades de informação que compõem o Sistema de Bibliotecas da UFSC.

O universo populacional da presente pesquisa foi constituído de 1.053 pesquisadores-docentes, dos 50 programas de pós-graduação da Universidade Federal de Santa Catarina (UFSC). A amostra foi constituída de 290 pesquisadores, e determinada por meio das fórmulas sugeridas por Barbetta (2002), considerando-se um erro amostral de 5\%. A ela foi acrescido, aproximadamente, $10 \%$ no número de pesquisadores, para garantir um maior índice de respostas. No final da fase de tabulação, a 
composição da amostra para esta pesquisa ficou constituída por 324 respondentes. Para obter uma amostragem que fosse considerada representativa dos pesquisadores, optou-se por um processo de seleção que considerou as áreas de conhecimento - Ciências Exatas e da Terra, Ciências Humanas e Sociais e Ciências da Vida - classificação esta adotada nos anais da Semana de Ensino, Pesquisa e Extensão da UFSC (SEPEX) (SEMANA..., 2000). Para tanto, na primeira etapa a seleção foi de forma aleatória estratificada e proporcional, considerando-se as áreas citadas acima. Na segunda etapa, foi realizada de forma aleatória simples, considerando-se os conceitos (3, 4, 5, 6 e 7) obtidos na avaliação da Capes. Na última etapa, para a definição final da amostra, a seleção foi de forma aleatória por conglomerados. O conceito obtido na avaliação da Capes foi considerado como indicador para a seleção da amostra deste estudo, uma vez que ele sinaliza os cursos que dispõem de melhor estruturação, tanto com relação aos recursos humanos, quanto com relação à produtividade dos docentes vinculados aos programas de pósgraduação das instituições de ensino superior do Brasil. Foram excluídos da amostra: pesquisadores-docentes que não eram do quadro permanente da UFSC (professores visitantes; participantes de outras instituições); pesquisadores-docentes que não estavam credenciados nos programas de pós-graduação da UFSC; pesquisadores-docentes que se recusaram a participar da pesquisa; pesquisadores-docentes que estavam afastados para formação, ou por licença para tratamento de saúde.

Para a coleta de dados foi utilizado como instrumento um questionário enviado como anexo via e-mail ou entregue impresso, acompanhado de uma carta de apresentação. O questionário foi adotado por se constituir, segundo Labes (1998, p. 16), uma grande fonte de fidedignidade, "pois não se discute o que foi formalmente registrado, afastando-se, assim, a velha crítica da validade dos resultados de uma pesquisa". Gil (2002) indica que o questionário, além de ser um instrumento de coleta de dados mais rápido e barato, e de não exigir treinamentos para sua aplicação, também garante o anonimato do respondente. $O$ instrumento apresentou 20 questões fechadas e semiabertas, que foram divididas em dois blocos, descritos a seguir, para facilitar a operacionalização da análise das variáveis.

1. Bloco 1 - Caracterização dos Participantes: Compreendeu as questões referentes à identificação dos respondentes quanto às características sociodemográficas e acadêmicas, tais como: sexo, faixa etária, regime de trabalho, formação acadêmica, nome do programa de pós-graduação e participação em grupos de pesquisa.

2. Bloco 2 - Uso dos recursos informacionais: Neste bloco, as questões diziam respeito diretamente ao processo de busca da informação. Foram então incluídas questões referentes ao uso dos recursos informacionais (on-line ou não) utilizados para satisfazer as necessidades de informação dos pesquisadores, bem como questões que pudessem indicar a freqüência de uso 
do Sistema de Bibliotecas da UFSC, freqüência de uso da Internet, barreiras encontradas para o uso da Internet, finalidades de uso da Internet, preferências de formato dos recursos informacionais, motivos que justificassem o formato preferido. Foram incluídas questões específicas referentes à intermediação no processo de busca da informação, para reforçar a avaliação dos resultados obtidos quanto à existência do fenômeno de desintermediação da informação, possivelmente detectados nos demais itens do questionário.

Os dados obtidos foram digitados numa planilha específica do Statistical Package for the Social Sciences (SPSS), versão 11.0 para Windows. Os dados quantitativos foram analisados por meio da estatística descritiva (freqüências e porcentagens). Para analisar as associações entre as áreas de atuação, a faixa etária, o regime de trabalho dos docentes e o uso dos recursos eletrônicos, utilizou-se o teste de Quiquadrado (n.2). As variáveis referentes às questões destinadas a verificar a ocorrência da desintermediação entre as áreas de atuação dos docentes foram analisadas utilizando-se o teste de Kruskal-Wallis, por área de atuação; sendo que, nas variáveis em que ocorreram diferenças estatisticamente significativas, foi aplicado o teste de Mann-Whitney para detectar entre quais áreas elas ocorreram (SIEGEL, 1977). O teste Kruskal-Wallis é um teste estatístico não paramétrico, que permite comparar mais de duas amostras de população independentes, enquanto que o teste de Mann-Whitney identifica as diferenças ocorridas entre as amostras independentes (SIEGEL, 1977).

Em todas as análises estatísticas consideraram-se, como diferenças significativas, os resultados com p 0,05.

\section{Resultados: descrição e análise}

Seguindo os objetivos que nortearam este trabalho, primeiramente serão apresentadas as análises descritivas quanto ao perfil sociodemográfico dos pesquisadores, aos percursos realizados para a obtenção da informação, aos recursos eletrônicos mais utilizados, à freqüência e às barreiras para o uso da internet. Em seguida, serão apresentadas as análises de associação das características do perfil dos pesquisadores com o uso de recursos eletrônicos e, por último, as análises referentes à ocorrência da desintermediação no processo de busca da informação, nas áreas de atuação dos pesquisadores.

a) Quanto à caracterização dos pesquisadores: Observou-se que $66,7 \%$ dos pesquisadores eram do sexo masculino e que, aproximadamente, um em cada dois pesquisadores tinha entre 40 e 49 anos. Quanto ao regime de trabalho, 89,5\% atuavam em regime de tempo integral, com dedicação exclusiva. Com relação à formação 
acadêmica, todos eram doutores, com uma predominância do período de capacitação entre os anos 1990 a 1999;

b) Quanto ao percurso utilizado pelos pesquisadores para a obtenção da informação: A biblioteca particular foi apontada pelos pesquisadores como primeira opção no percurso escolhido para o processo de busca da informação. No entanto, quando considerada a soma das três primeiras opções, a Internet obteve o maior índice de escolha, seguida da biblioteca particular e das bibliotecas centrais e setoriais. A acessibilidade (proximidade física e disponibilidade de equipamentos) para o desempenho de tarefas ou atividades desenvolvidas pelos pesquisadores foi considerada como um dos fatores associados ao uso dessas duas opções de percurso (Internet e biblioteca particular). O baixo índice de uso do Sistema BU/UFSC pode ser atribuído aos parcos investimentos do governo federal destinados à melhoria das coleções locais das bibliotecas universitárias brasileiras, a problemas relacionados ao atendimento pessoal e à qualidade e atualidade do acervo;

c) Quanto à freqüência de uso do Sistema BU/UFSC: Detectou-se que, embora existisse uma freqüência de uso diário muito baixa do Sistema BU/UFSC, ao se analisar em conjunto o uso diário, semanal e mensal, detectou-se uma modificação expressiva nesse resultado, pois $77,8 \%$ dos pesquisadores indicaram uma freqüência considerada significativa, em comparação com os pesquisadores que relataram um uso esporádico do Sistema BU/UFSC, que somam 22,2\% do total. Considerou-se que, embora a BU/UFSC não tenha sido escolhida como a primeira opção no percurso de busca da informação pelos pesquisadores, a mesma apresentou um índice de freqüência bastante relevante. Sendo assim, esse resultado pode ter sido influenciado pelo fator utilidade, uma vez que, dependendo da atividade em que o pesquisador estiver atuando (pesquisa, ensino ou administração), ele poderá, de alguma forma, vir a adotar esse percurso, fazer uso de seus recursos informacionais disponíveis e considerá-los importantes para suas necessidades naquele momento;

d) Quanto à biblioteca e seus recursos mais utilizados: Os periódicos foram os recursos mais utilizados, seguido dos livros e das bases de dados. Nesse sentido, constata-se que, embora os recursos informacionais em formato eletrônico já estejam fazendo parte das atividades de busca de informações desses pesquisadores, eles ainda utilizam os recursos informacionais tradicionais disponibilizados pelo Sistema BU/UFSC;

e) Quanto ao formato preferido dos recursos informacionais: Dos 324 pesquisadores, $51,5 \%$ preferiam utilizar recursos informacionais no formato eletrônico, enquanto que $44,1 \%$ tinham preferência pelo formato impresso e 4,4\% por ambos. Percebeu-se que, embora exista preferência pelo formato eletrônico, o formato impresso obteve um índice significativo. Pode-se considerar, portanto, que a comunicação formal ainda esteja passando por um período de transição do formato impresso para o totalmente eletrônico, e isso implica a convivência, por algum tempo, com essas duas opções; 
f) Quanto aos motivos da preferência do formato: A acessibilidade, contabilizando $46,9 \%$ das incidências nas indicações dos pesquisadores, foi o principal motivo para a escolha do formato dos recursos informacionais, seguido da praticidade e da agilidade. Nesse sentido, considera-se que os fatores de ordem pessoal sejam os principais responsáveis pela escolha de um ou de outro formato;

g) Quanto ao processo de busca da informação - mediadores preferenciais: Os colegas foram indicados como primeira opção, seguidos por colegas de universidades brasileiras e estrangeiras. Os bibliotecários obtiveram um índice inexpressivo de indicações, o que demonstrou que eles não foram considerados por essa comunidade como mediadores preferenciais no processo de busca da informação. Esses resultados levam a constatar que as unidades de informação têm proporcionado, por meio da disponibilização de recursos informacionais em formato eletrônico, serviços on-line e treinamentos virtuais ou presenciais; o que leva seus usuários a uma certa autonomia no processo de busca da informação. Isso nos leva a acreditar que os bibliotecários, profissionais atuantes nessas unidades de informação, não sejam considerados por essa comunidade como mediadores, pensando em mediação face-a-face. Entretanto, esses profissionais estão possibilitando formas inovadoras de mediação, a partir do momento em que passaram a atuar na orientação do uso das TICs, na seleção de conteúdos de informação, no desenvolvimento de sistemas especialistas para atender às questões de referência, na capacitação de seus usuários e em tantas outras tarefas decorrentes da introdução das TICs nas unidades de informação e na vida dos pesquisadores;

h) Quanto aos recursos informacionais indispensáveis: Observou-se que os recursos mais indicados foram as bases de dados referenciais/texto completo, seguidas dos portais de pesquisa. Aqui, mais uma vez, a importância do acesso à informação on-line destaca-se na preferência dessa comunidade. Sem dúvida, a recuperação eletrônica permitiu aos pesquisadores maior qualidade nas suas pesquisas, e a Internet veio facilitar ainda mais o processo de busca da informação;

i) Quanto aos recursos da Internet: O e-mail foi o recurso mais utilizado, dentre os demais recursos da Internet, seguido da consulta a revistas eletrônicas e bases de dados. Grupos de discussão e outros recursos da rede obtiveram um índice relativamente baixo. Esses resultados demonstram que os principais motivos para a utilização da rede são a comunicação interpessoal e a obtenção da informação por meio eletrônico. Os demais recursos são pouco utilizados ou desconhecidos por essa comunidade. Nesse sentido, os resultados demonstram a necessidade de maior divulgação e incentivo, quanto aos recursos pouco utilizados por essa comunidade cientifica;

j) Quanto à finalidade de uso da Internet: Dos 324 pesquisadores, $65,4 \%$ confirmaram que usam a rede para fins de comunicação. Em seguida, o levantamento bibliográfico foi indicado por $22,2 \%$ dos pesquisadores. A circulação de trabalhos antes da publicação e a submissão de artigos a periódicos e eventos obtiveram índices inexpressivos. O uso da Internet no processo de comunicação científica 
ainda é muito vinculado à comunicação informal, a qual vem possibilitando mais agilidade no processo de comunicação entre os pares, oferecendo maiores oportunidades para a formação de colégios invisíveis;

k) Quanto à freqüência de uso da Internet: De modo geral, a Internet tem sido usada por quase todos os respondentes. O uso diário foi apontado por $81,8 \%$ dos pesquisadores. Quando analisadas conjuntamente as freqüências (diária, semanal e mensal), esse resultado sobe para 99,1\%. Nesta pesquisa, detectou-se que apenas 0,9\% dos pesquisadores não utilizam a Internet;

I) Quanto ao uso dos serviços on-line do Sistema BU/UFSC: Dentre os serviços on-line oferecidos pelo Sistema BU/UFSC, o Portal Capes foi o recurso mais utilizado pelos pesquisadores, seguido do sistema de consulta Pergamum. Cabe ressaltar que alguns serviços obtiveram índices relativamente baixos, como é o caso do Portal de Pesquisa (DotLib), indicado por $0,6 \%$ dos pesquisadores. Esse fato pode estar associado ao desconhecimento dos pesquisadores sobre alguns serviços disponibilizados pelo Sistema BU/UFSC;

m) Quanto ao uso das bases de dados do Portal Capes: Observou-se uma predominância no uso das bases de dados Web of Science do ISI (Institute for Scientific Information), com 49 indicações. Outras Bases de dados, como Elsevier/Science Direct, Medline e Scielo, também obtiveram indicações expressivas;

n) Quanto às barreiras para uso da Internet: A maior dificuldade encontrada para o uso da Internet, na UFSC, está relacionada à infraestrutura. Dos 324 respondentes, 33,3\% indicaram problemas de conexão, seguidos da falta de suporte técnico, com 21,3\%. De maneira geral, esses resultados podem ser considerados comuns aos ambientes de rede, tanto nacionais quanto internacionais. Fatores relacionados a questões pessoais, tais como a habilidade no uso de tecnologias e lingüística, foram praticamente desconsiderados por essa comunidade;

o) Quanto às associações entre o perfil dos pesquisadores e uso de recursos eletrônicos: Não foram observadas associações estatisticamente significativas entre a utilização da Internet, como opção de percurso de informação preferido, e a faixa etária dos pesquisadores, regime de trabalho e área de atuação. No entanto, quando da realização do teste de n.2 entre as barreiras para o uso da Internet e a variável área de atuação,percebeu-se que ocorreram algumas associações estatisticamente significativas. Ao analisar esses resultados, percebe-se que, embora a freqüência de uso da rede seja representativa, existem algumas barreiras que podem influenciar a eficácia de seu uso. Os resultados demonstraram que os pesquisadores da área de Ciências Humanas e Sociais têm enfrentado problemas relativos ao uso da Internet e que estes estão relacionados com problemas de infra-estrutura (falta de equipamento e ausência de suporte técnico). Nas associações entre a utilização dos recursos eletrônicos da Internet (grupos de discussão, e-mail, revistas eletrônicas, bases de dados com texto completo) e as áreas de atuação, não foram observadas associações estatisticamente significativas. Entretanto, foram encontradas associações estatisticamente significativas 
entre o uso de recurso eletrônico relacionado às bases de dados referenciais e a área de atuação. Na área de Ciências da Vida, ocorreu uma incidência de $64,3 \%$ entre os pesquisadores que utilizam as bases de dados referenciais, considerando-se a soma das três opções na indicação de uso para esse recurso. Não foram observadas associações estatisticamente significativas entre o uso dos recursos informacionais online, disponibilizados pelo Sistema de Bibliotecas da UFSC (Catálogo online - Pergamum, Portal Capes, Portal da Pesquisa - DotLib, reserva de livros, renovação de empréstimo, lista de periódicos on-line) e as áreas de atuação. Apenas, como já foi observado anteriormente, o uso de bases de dados referenciais foi mais incidente na área de Ciências da Vida;

p) Quanto à ocorrência da desintermediação da informação no processo de busca da informação entre as áreas de atuação dos pesquisadores:

- Quanto ao percurso preferido no processo de busca da informação: O teste de Kruskal-Wallis não detectou diferenças significativas entre as áreas de atuação (Ciências Exatas e da Terra, Ciências Humanas e Sociais e Ciências da Vida) quanto à utilização dos percursos no processo de busca da informação com maior freqüência de uso (Internet, biblioteca central e biblioteca particular). No entanto, detectou diferenças estatisticamente significativas entre as áreas quanto ao uso de bibliotecas setoriais. Verificou-se um uso mais freqüente da biblioteca setorial entre os pesquisadores da área de Exatas e da Terra;

- Quanto à freqüência de uso do Sistema BU/UFSC: Não foram observadas diferenças significativas entre as áreas de atuação (Ciências Exatas e da Terra, Ciências Humanas e Sociais e Ciências da Vida) quanto à freqüência de uso do Sistema BU/UFSC. Todas as áreas têm utilizado o Sistema de BU/UFSC na mesma proporção;

- Quanto ao uso dos recursos informacionais do Sistema BU/UFSC: O teste de Kruskal-Wallis não detectou diferenças significativas entre as áreas de atuação (Ciências Exatas e da Terra, Ciências Humanas e Sociais e Ciências da Vida) quanto à utilização dos recursos informacionais disponibilizados pelo Sistema BU/UFSC (livros, periódicos, bases de dados, Comutação, normas técnicas) Entretanto, detectou diferenças estatisticamente significativas entre as áreas quanto ao uso de dicionários do Sistema BU/UFSC. Verificou-se um uso mais freqüente desse recurso entre os pesquisadores da área de Ciências Humanas e Sociais;

- Quanto aos recursos informacionais on-line do Sistema BU/UFSC: O teste de Kruskal-Wallis não detectou diferenças significativas entre as áreas de atuação (Ciências Exatas e da Terra, Ciências Humanas e Sociais e Ciências da Vida) quanto à utilização dos recursos informacionais on-line disponibilizados pelo Sistema BU/UFSC (Portal Capes, Portal da Pesquisa (DotLib), reserva de livros, lista de periódicos on-line). Entretanto, detectou diferenças estatisticamente significativas entre as áreas quanto ao uso do Catálogo on-line Pergamum, serviço de renovação de empréstimo e bases de dados referenciais. Quanto ao uso do Catálogo Pergamum, verificou-se maior incidência de uso entre os pesquisadores da área de Ciências Humanas e Sociais; 
- Quanto a quem recorrer na busca da informação: Não foram observadas diferenças significativas entre as áreas de atuação (Ciências Exatas e da Terra, Ciências Humanas e Sociais e Ciências da Vida) quanto a quem recorrer na busca da informação (colegas pares/grupo pesquisa, colegas de universidades brasileiras). Contudo, detectaram-se diferenças estatisticamente significativas entre as áreas quanto ao auxílio de colegas de universidades estrangeiras e de profissionais bibliotecários. Quanto à busca de auxílio dos profissionais bibliotecários, os pesquisadores da área de Ciências Humanas e Sociais indicaram que solicitavam mais a ajuda desses profissionais, no processo de busca da informação.

\section{Conclusão}

Finalmente, os resultados desta pesquisa, sobre o uso da Internet no processo de busca da informação pelos pesquisadores da UFSC, mostraram que a rede tem afetado significativamente o processo de comunicação científica, sob dois aspectos: a comunicação informal e o acesso à informação. A comunicação informal mediada por computador já é uma realidade nas comunidades científicas, em todo o mundo. 0 processo de busca da informação de forma on-line garante a rapidez na obtenção de resultados, elimina barreiras de espaço e tempo, diminui a dependência dos intermediários, sejam eles instituições ou pessoas.

Quanto ao fenômeno de desintermediação da informação, conclui-se que está ocorrendo um aumento do mesmo em todas as áreas, uma vez que os pesquisadores estão utilizando significativamente os recursos informacionais em formato eletrônico, no processo de busca da informação. Contudo, tendo em vista o uso expressivo, pelos pesquisadores desta investigação, do acervo tradicional, bem como dos serviços on-line disponibilizados pelo Sistema BU/UFSC, considera-se que ainda existe dependência no processo de busca da informação, em relação à instituição biblioteca (unidade de informação), sendo esta a principal provedora de recursos de informação, tanto eletrônicos quanto tradicionais.

Quanto aos profissionais bibliotecários, os resultados permitiram concluir que os mesmos não são os mediadores da informação preferidos por essa comunidade científica. Assim, considerando-se que esses profissionais têm trabalhado no sentido de aumentar os recursos on-line para pesquisa disponibilizados nas universidades, como também têm investido esforços no treinamento para tornar os usuários mais autônomos, o que se pode inferir é que provavelmente a desintermediação tenha ocorrido como conseqüência natural dessas ações. Na verdade, considerou-se que a forma de mediação desses profissionais tenha mudado de foco, como sinal da evolução ou da crise dos sistemas de informação que, na atualidade, dependem muito mais de serviços de organização e acesso à informação e de interfaces amigáveis que facilitem a busca, a localização e a recuperação, do que dos próprios recursos humanos e físicos existentes nas unidades de informação. 
Com base nos resultados alcançados nesta pesquisa, podem ser apresentadas algumas reflexões a respeito do uso da informação, do papel das unidades de informação e dos mediadores da informação.

O uso das TICs faz parte do contexto de pesquisa das comunidades científicas em geral e especificamente nessa comunidade de pesquisadores da UFSC. As bibliotecas e seus profissionais, por isso, devem sair da defensiva e inovar suas formas de disseminação da informação, para atender às novas exigências e expectativas dos usuários de redes de informação.

Posto isso, cada vez mais programas de divulgação e treinamento são fundamentais para otimizar o uso das TICs, não só para facilitar o acesso ao manancial de informações disponibilizadas pela Internet, mas, sobretudo, para tornar os indivíduos competentes em informação. A função da biblioteca, juntamente com seus profissionais, é de criar novas formas de mediação, no sentido de orientar e estimular as competências dos usuários na identificação de seus problemas/necessidades de informação, no acesso, na avaliação e no uso das informações disponíveis na Internet. Nesse sentido, acredita-se que a mediação humana ainda seja insubstituível, pois a mediação puramente eletrônica, proporcionada pelas ferramentas de busca de informação, apesar de seus avanços, não consegue substituir totalmente as competências de um profissional de informação capacitado para suas novas funções.

$\mathrm{Na}$ realidade, os profissionais deverão utilizar-se das vantagens oferecidas pelas TICs em beneficio próprio, criando assim novas formas de mediação. Um exemplo disso seria a preocupação com a concepção de ferramentas de interface mais amigáveis para os sites das unidades de informação, para que possam atender às diferentes necessidades dos usuários, com diferentes níveis de experiência e competência na utilização de recursos informacionais eletrônicos e técnicas de pesquisa.

As bibliotecas deverão atuar como catalisadoras dos recursos informacionais dispersos pela rede, realizando suas tarefas já tradicionalmente conhecidas, que são a de selecionar, organizar e disseminar os recursos relevantes disponibilizados também pela rede.

Para Fourie (2001), o processo de desintermediação poderá ser considerado como uma oportunidade para os profissionais da informação, uma vez que, estando os usuários finais aptos a assumir as suas próprias buscas de informação, a atenção desses profissionais e das instituições poderá estar voltada para outras questões também importantes; o que poderá desencadear uma reflexão crítica, um refinamento das habilidades existentes e a expansão continuada de outras.

No que se refere ao processo de busca da informação, nesta pesquisa foi detectado o quanto ainda são importantes os recursos informacionais impressos, para os pesquisadores. Sem deixarmos de considerar que as TICs trouxeram avanços fundamentais, tanto para os usuários como para as próprias unidades de informação, ainda assim os documentos impressos têm seu espaço garantido, pois representam uma forma expressiva e respeitável de disseminação de informações. Por isso, acredita-se que coexistirão por muito tempo os meios impressos e 
eletrônicos, e que nem todos os usuários desejarão e poderão usar os recursos eletrônicos. Assim, é fundamental que as bibliotecas flexibilizem seus acervos e serviços, para poderem atender os diferentes usuários e suas diferentes necessidades de informação.

Nesse sentido, o conceito de biblioteca híbrida parece ser bem indicado para atender às necessidades informacionais atuais, neste momento de transição pelo qual as unidades de informação convencionais estão passando. Isto é, para atender de forma efetiva seus usuários, as bibliotecas não poderão ter somente coleções impressas ou digitais, deverão ter coleções que contemplem os diversos formatos nos quais as informações estão disponibilizadas. Caberá também às unidades de informação um desafio muito grande, com a finalidade de prover o acesso à informação impressa, ainda necessária para as comunidades científicas, como também o acesso à informação eletrônica, já que grande parte das informações de pesquisa de ponta ainda já se encontra nesse formato.

A busca de parcerias e consórcios, em um país como o Brasil, para suprir deficiências geradas pela carência de investimentos nesse setor, parece ser uma alternativa viável neste momento de transição. Um outro aspecto, que não deve ser deixado de lado, está relacionado à infraestrutura para o acesso à informação através das redes existentes nas universidades. Para que as bibliotecas possam disponibilizar cada vez mais recursos informacionais eletrônicos, é imprescindível que se façam investimentos em infra-estrutura de rede, provendo maior velocidade de tráfego nas redes de comunicação, aprimorando as arquiteturas, topologias e protocolos de rede, para a otimização de seu uso em ambientes distribuídos, entre outros, que são condições fundamentais e necessárias para a implantação de bibliotecas virtuais e/ou digitais.

\section{Referências}

ABELS, E. G.; LIEBSCHER, P.; DENMAN, D. Factors that influence the use of electronic network by science and engineering faculty at small institutions. part I. Queries. Journal of the American Society for Information Science, v. 47, n. 2, p. 146-158, 1996.

BARBETTA, P. A. Estatística aplicada às ciências sociais. 5. ed. rev. Florianópolis: Ed. da UFSC, 2002.

BROWN, J. S.; DUGUID, P. A vida social da informação. São Paulo: Makron Books, c2001.

CASTELLANI, M. R.; ZWICKER, R. Informatizando a comunicação na universidade; uma análise cultural. Revista de Administração, São Paulo, v. 35, n. 2, p. 10-18, abr./jun. 2000.

COSTA, S. M. S.; SILVA, W. A. A da; COSTA, M. B. Publicações científicas eletrônicas no Brasil: mudanças na comunicação formal, também? Revista de Biblioteconomia de Brasília, Brasília, v. 25, n. 1, p. 57-76, jan./jun. 2001. 
CONFERENCE ON OPEN ACCESS TO KNOWLEDGE IN THE SCIENCES AND HUMANITIES, 2003, Berlin. Press release. Berlin, 2003. Disponível em: <http:// www.zim.mpg.de/openaccess-berlin/pressstatement. html>. Acesso em: 30 jun.2004.

FERREIRA, S. S. P. Redes eletrônicas e necessidades de informação: abordagem do "sense making" para estudo de comportamento de usuários do instituto de Física da USP. 1995, 165 f. Tese (Doutorado, em Ciências Comunicações) - Escola de Comunicações e Artes, USP, São Paulo, 1995.

FOURIE, I. Debemos tomarnos en serio la desintermediación? Anales de Documentación: Revista de Biblioteconomia e Documentación, Murcia, v.4, p.267-282, 2001. Disponível em: <http://www.um.es/fccd/anales/ad04/ ad0400.html>. Acesso em: 12 jun. 2004.

GARVEY, W. D. Communication: the essence of science. Oxford: Pergamon, 1979.

GIL, A. C. Como elaborar projetos de pesquisa. 4. ed. São Paulo: Atlas, 2002.

KUHN, T. S. A estrutura das revoluções científicas. 3. ed. São Paulo: Perspectiva, 1992.

LABES, E. M. Questionário: do planejamento à aplicação na pesquisa. Chapecó: Grifos, 1998.

LOVISOLO, H. Comunidades científicas: condições ou estratégias de mudança. Educação \& Sociedade, Campinas, v. 18, n. 59, p. 270-297, ago. 1997.

MEADOWS, A. J. A comunicação científica. Brasília: Briquet Lemos, 1999.

- Periódicos científicos, do meio impresso para o eletrônico. Revista de Biblioteconomia de Brasília, Brasília, v. 25, n. 1, p. 5-14, jan./jun. 2001.

NEHMY, R. M. Q.; PAIM, I. O uso de informações na Internet: estudo de caso de um fórum sobre gestão do conhecimento. In: ENCONTRO NACIONAL DE PESQUISA EM CIÊNCIA DA INFORMAÇÃO, 5., 2003, Belo Horizonte. Anais... Belo Horizonte: Escola de Ciência da Informação da UFMG, 2003.

PINHEIRO, L. N. R. Comunidades científicas e infra-estrutura tecnológica no Brasil para uso de recursos eletrônicos de comunicação e informação na pesquisa. Ciência da Informação, Brasília, v. 32, n. 3, p.62-73, set./dez. 2003.

REDE NACIONAL DE PESQUISA (Brasil). Sobre a Internet. Brasília, 2005. Disponível em: <http://www.rnp.br/ rnp>. Acesso em: 23 abr. 2005.

RICHARDSON, R. J. et al. Pesquisa social: métodos e técnicas. 3. ed. rev. ampl. São Paulo: Atlas, 1999.

SEMANA NACIONAL DE ENSINO, PESQUISA E EXTENSÃO, 1.,2000, Florianópolis. Anais... Florianópolis: UFSC, 2000. 
SIEGEL, S. Estatística não paramétrica. São Paulo: McGraw Hill do Brasil, 1977.

SILVA, E. L. da; MENEZES, E. M.; BISSANI, M. A Internet como canal de comunicação científica. Informação \& Sociedade: Estudos, João Pessoa, v. 12, n. 1, 2002 Disponível em: <http://www.informacaoesociedade.ufpb.br/ 1210212.html >. Acesso em: 23 mar. 2004.

SOLLA PRICE, D. J. de. O desenvolvimento da ciência: análise histórica, filosófica, sociológica e econômica. Rio de Janeiro: Livros Técnicos e Científicos, 1976.

SOUZA, M. da P. N. de. Efeitos das tecnologias da informação na comunicação de pesquisadores da Embrapa. Ciência da Informação, Brasília, v. 32, n. 1, p. 135-143, jan./abr. 2003.

SULLIVAN, P. M. A. Sobre las bibliotecas, los bibliotecarios y la globalización. Lima: Universidade de Buenos Aires, jul. 2002. Disponivel em: <http://eprints.rclis.org/archive/00002328/01/B13-01.pdf >. Acesso em: 5 maio 2005.

TARAPANOFF, K.; ARAÚJO JÚNIOR, R. H. de; CORMIER, P. M. J. Sociedade da informação e inteligência em unidades de informação. Ciência da Informação, Brasília, v. 29, n. 3, p. 91-100, set./dez. 2000.

TARGINO, M. das G. Divulgação de resultados como expressão da função social do pesquisador. Revista de Biblioteconomia de Brasília, Brasília, v. 23/24, n. 3, p. 347-366, 1999/2000.

TARGINO, M. das G. Comunicação científica: uma revisão de seus elementos básicos. Informação \& Sociedade: Estudos, João Pessoa, v. 10, n. 2, jul./dez. 2000. Disponível em: <http://www.informacaoesociedade.ufpb.br/1020002.pdf $>$. Acesso em: 12 mar. 2004.

Novas tecnologias e produção científica: uma relação de causa e efeito ou uma relação de muitos efeitos? In: SEMINARIO DE BIBLIOTECAS UNIVERSITÁRIAS, 12., 2002, Recife. Anais... Recife: Dot Lib, 2002. 1 CDRom.

VAZ, P. Mediação e tecnologia. Revista da FAMECOS, Porto Alegre, v.16, n.1, p.45-58, 2001. Disponível em: <http://www.eco.ufri/ciberidia/equipe/pdf/ paulovaz/Midia/E30e tcnologiarevista.pdf>. Acesso em: 29 abr. 2005.

VIEIRA, J. L. G. Correio eletrônico: o impacto na comunicação técnicocientífica da EMBRAPA: estudo de caso. 1998, 158f. Dissertação (Mestrado em Ciência da Informação) - Escola de Comunicação, Universidade Federal do Rio de Janeiro, Rio de Janeiro, 1998.

ZIMAN, J. Conhecimento público. São Paulo, Itatiaia, 1979. 